\title{
Nanodomains in morphotropic lead zirconate titanate ceramics: On the origin of the strong piezoelectric effect
}

\author{
Ralf Theissmann, ${ }^{a}$ Ljubomira A. Schmitt, Jens Kling, Roland Schierholz, \\ Kristin A. Schönau, and Hartmut Fuess \\ Institute of Material Science, Darmstadt University of Technology, 64287 Darmstadt, Germany \\ Michael Knapp \\ CELLS, P.O. Box 68, 08193 Barcelona, Spain \\ Hans Kungl and Michael J. Hoffmann \\ Institute of Ceramics in Mechanical Engineering, University of Karlsruhe, 76131 Karlsruhe, Germany
}

(Received 14 March 2007; accepted 29 May 2007; published online 23 July 2007)

\begin{abstract}
The outstanding piezoelectric properties of lead zirconate titanate (PZT) ceramics with compositions close to the morphotropic phase boundary of the quasibinary phase diagram of lead zirconate and lead titanate are still under debate. A combination of ex situ and in situ transmission electron microscopy and high resolution x-ray diffraction revealed that the extrinsic piezoelectric effect in morphotropic PZT is closely connected to the existence of nanodomains. The in situ transmission electron microscopy investigations with applied electric field show that mainly the nanodomains respond to the electric field while the microdomain structure does not change noticeably in our experiments. (C) 2007 American Institute of Physics. [DOI: 10.1063/1.2753569]
\end{abstract}

\section{INTRODUCTION}

The ability of a piezoelectric material to translate mechanical pressure into an electric signal or vice versa has already been used in a variety of important applications. Ceramics containing mixed crystals of lead zirconate and titanate, $\mathrm{PbZr}_{1-x} \mathrm{Ti}_{x} \mathrm{O}_{3}(\mathrm{PZT})$, are the most important representatives of piezoelectric materials because of their outstanding performance at a composition of approximately half zirconate and half titanate. Such ceramics combine a very strong piezoelectric effect with a high remnant polarization, low switching currents and fast switching times, which can be tuned by composition and the addition of dopants.

Although PZT ceramics have been established in technical applications for years, the microstructure of this class of materials is still under investigation and the atomistic phenomena that cause the strong extrinsic piezoelectric and ferroelectric effect are not yet understood, neither the mechanisms of its degradation.

So far, the microstructure of PZTs close to the morphotropic phase boundary (MPB) of the quasibinary phase diagram of lead zirconate and lead titanate (Fig. 1) has not been completely elucidated. This is mainly due to the decrease of domain widths for compositions in the MPB region. Domain sizes of less than $100 \mathrm{~nm}$ are reported for different compositions and doping levels. ${ }^{1-9}$ The investigation of the crystallographic structure of a single domain by conventional selected area electron diffraction is limited by the small volumes under study. Furthermore, the existence of nanodomains, which are defined as nm-sized domains within microdomains (definition introduced by Schmitt et al. ${ }^{10}$ ),

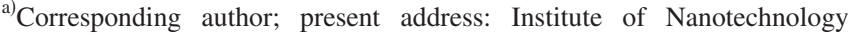
Forschungszentrum Karlsruhe, P.O. Box 3640, 76021 Karlsruhe, Germany; electronic mail: ralf.theissmann@int.fzk.de
}

complicates the interpretation of conventional x-ray experiments because the coherent scattering of the nanodomains and/or domain walls contributes to the observed diffraction patterns. ${ }^{5-7,11-16}$ A misinterpretation of the refined structural model might be the result.

Several theories have been introduced to explain seemingly conflicting experimental evidence. The contribution of the extrinsic ferroelectric effect to the polarization of the ferroelectric ceramics is generally attributed to an increasing volume fraction of material that is polarized in the direction of an external electric field. The mechanisms, which lead to

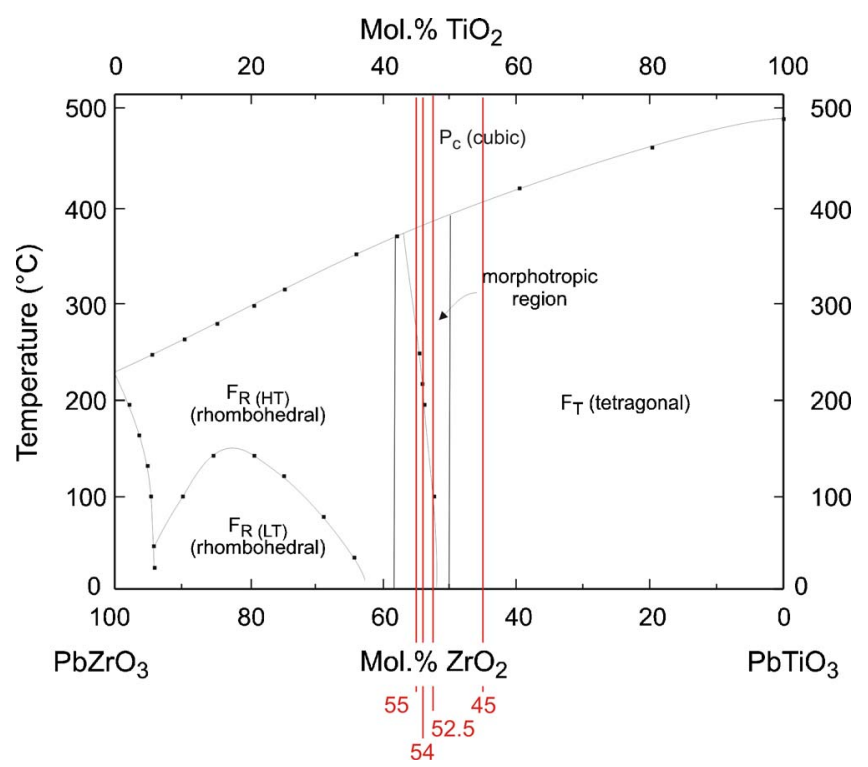

FIG. 1. (Color online) Phase diagram of the $\mathrm{PbZrO}_{3}-\mathrm{PbTiO}_{3}$ system with the compositions of the investigated samples marked. Phase diagram can be found, e.g., in Ref. 23. 
the extraordinary piezoelectric properties, are commonly discussed in terms of domain-wall motion or formation of transient domain walls.

Based on these assumptions, a number of theories can be found to explain the behavior of ferroelectric PZT ceramics:

(a) The increase of the dielectric constant near the morphotropic phase boundary is explained by the coexistence of the tetragonal and the rhombohedral crystal structure $^{11}$ with six and eight equivalent polarization directions, respectively. It is argued that the coexistence of the two polymorphs leads to 14 possible polarization directions and therefore 14 directions into which the material can be polarized under the influence of an external electric field.

(b) The existence of a monoclinic phase in the region of the MPB was suggested by Noheda et al. ${ }^{12}$ The Rietveld refinement of the structural model based on $\mathrm{x}$-ray full-pattern data showed that the introduction of this phase improved the agreement between experimental and calculated data significantly. Furthermore, it introduces additional directions for the polarization, which can explain the outstanding ferroelectric properties similar to model (a).

(c) In general, Jin et al. ${ }^{8,17}$ showed later that coherently scattering tetragonal nanometer-sized domains give rise to x-ray patterns, which can be indexed as monoclinic. Their calculations revealed a structural model for the so called "adaptive phase," which can also be extended to PZT. The model was further developed by the theoretical work of Wang ${ }^{15,18}$ concerning nanotwin superlattices.

We will use a combination of ex situ and in situ transmission electron microscopy (TEM) and high resolution $\mathrm{X}$-ray diffraction (XRD) to demonstrate that the extrinsic piezoelectric effect in morphotropic PZT is closely connected to the existence of nanodomains.

\section{EXPERIMENTAL}

Undoped PZT samples with 45, 52.5, 54, and 55 mol \% of $\mathrm{ZrO}_{2}$ were investigated. All samples were prepared via a ceramic route as polycrystalline pellets. ${ }^{19,20}$ The samples used for the ex situ investigations were sintered at $1225^{\circ} \mathrm{C}$; samples for the in situ and convergent beam electron diffraction (CBED) investigations at $1050{ }^{\circ} \mathrm{C}$ for practical reasons (sample preparation). No significant differences between samples of identical composition sintered at $1050^{\circ} \mathrm{C}$ and $1225{ }^{\circ} \mathrm{C}$ were observed in x-ray diffraction.

Sample preparation for TEM investigations was done by subsequent cutting, polishing, dimpling, and ion milling. The microstructure of the samples is maintained by this preparation procedure. Investigations of the microstructure were performed along the three prominent axes [001], [011], [111]. Additionally, the symmetry of the samples was investigated by CBED along the [100] zone axis. The ex situ TEM investigation was carried out at a Philips CM20ST microscope, and in situ TEM studies at a FEI Tecnai F20 using a Gatan double tilt specimen holder with electric feedthrough. The sample was placed between two copper rings, which were connected to a high-voltage (HV) supply. The distance of the copper rings was $110 \mu \mathrm{m}$. The maximum applied voltage was $780 \mathrm{~V}(\max .7 \mathrm{kV} / \mathrm{mm})$. Calculations on the effective field distribution present at the investigated sample area are in progress.

High resolution $\mathrm{X}$-ray experiments were carried out at the beamline B2 at the HASYLAB (Hamburg, Germany). Disk shaped samples were cut and polished on both sides down to approximately $50 \mu \mathrm{m}$ and measured in transmission mode with a parallel incident beam and a scintillation detector. $^{21}$ Carefully chosen samples were measured in situ with an applied electric field. For this investigation, the samples were coated with platinum electrodes and placed in a specially designed sample holder in transmission geometry. Data collection was done with a stationary image plate system. ${ }^{22}$ The measuring time at each voltage was approximately $15 \mathrm{~min}$. Two doped samples with $52.5 \mathrm{~mol} \% \mathrm{ZrO}_{2}$ and $1 \mathrm{~mol} \% \mathrm{La}$ were prepared following the same synthesis route. The sintering temperature was $1050{ }^{\circ} \mathrm{C}$.

\section{RESULTS AND DISCUSSION}

We applied convergent beam electron diffraction to identify the crystal symmetry in the region of the morphotropic phase boundary with high precision. The crystallographic feature employed to distinguish the three polymorphs $(P 4 m m, C m, R 3 m)$ is the orientation of the mirror plane in the pseudocubic [100] projection whole patterns. In short, the loss of the parent cubic symmetry is accompanied by the disappearance of the fourfold axes in the rhombohedral case, whereas in the tetragonal case the threefold axes are lost. In both transitions different sets of mirror planes disappear. The remaining mirror planes in the pseudocubic [100] axiswhich is different from the tetragonal [001] axis-lie in the $\{100\}$ planes in the tetragonal case and in the $\{10-1\}$ planes for the rhombohedral case. For the monoclinic case no mirror plane is left, despite the $(-110)$ mirror if viewed along the formerly tetragonal [001] direction. To reliably distinguish the three phases, several neighboring domains were investigated in all occasions. Based on this method, the symmetry of representative domain configurations within samples containing up to $54 \mathrm{~mol} \%$ of $\mathrm{ZrO}_{2}$ were found to be tetragonal and the symmetry of representative domain configurations of samples with $55 \mathrm{~mol} \%$ (Fig. 2) and more $\mathrm{ZrO}_{2}$ (Ref. 10) is rhombohedral. The morphotropic phase boundary is therefore located between those compositions. Even if a possible coexistence of the tetragonal and rhombohedral polymorph cannot be completely excluded due to the limited number of examined specimens, one conclusion of our CBED results is that the ferroelectric properties are not dominated by the coexistence of the two phases. The limitation of the method was a minimum spot size of $12.5 \mathrm{~nm}$.

A series of samples across the morphotropic phase boundary with compositions indicated red within the phase diagram in Fig. 1 was investigated by conventional transmission electron microscopy and complementary x-ray diffraction. The joint interpretation of our detailed analysis of the microstructure leads to the suggestion of a model in which 


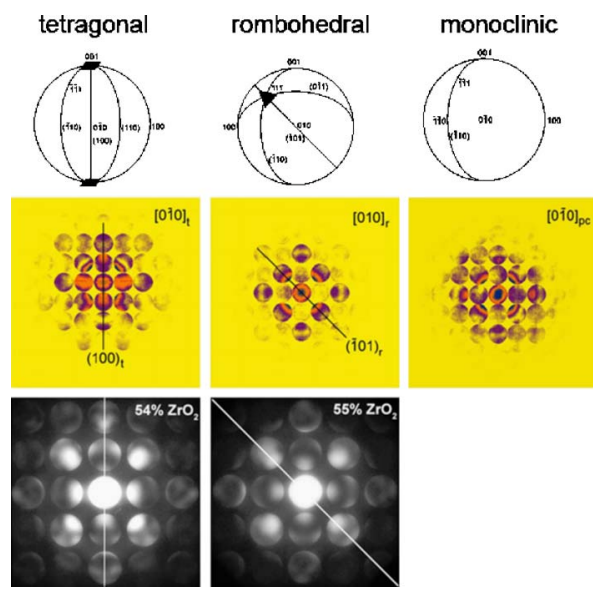

FIG. 2. (Color online) Stereographic projection for tetragonal, rhombohedral, and monoclinic symmetry (top row), simulated CBED patterns for the three symmetries (middle row), and experimental finding (bottom row): The sample containing $54 \%$ of $\mathrm{ZrO}_{2}$ shows tetragonal symmetry, the sample containing $55 \% \mathrm{ZrO}_{2}$ shows rhombohedral symmetry-mirror planes are indicated as a white line.

the extrinsic piezoelectric effect is closely connected to the existence of nanodomains. The presented data show that nanodomains are present only for compositions near the MPB. The width of the nanodomains rises toward the morphotropic composition approaching either from the zirconium rich side, with rhombohedral crystal structure, or from the titanium rich side, with tetragonal crystal structure of the microdomains. ${ }^{10}$ The identification of the nanodomains was performed by a set of TEM images along the [010] and [011] axes, as shown in Fig. 3. The lamellar microdomain structure of the sample containing $45 \mathrm{~mol} \%$ of $\mathrm{ZrO}_{2}$ is comparable to that previously reported in literature. ${ }^{24}$ As the composition approaches the morphotropic phase boundary, additional features are observed: The microdomain walls do not strictly obey the predicted crystallographic directions; bend microdomain walls are observed, most pronounced for the rhombohedral sample with $55 \mathrm{~mol} \% \mathrm{ZrO}_{2}$. In the samples with $52.5 \mathrm{~mol} \%$ and $54 \mathrm{~mol} \%$ of $\mathrm{ZrO}_{2}$ and the samples with $55 \mathrm{~mol} \%$ of $\mathrm{ZrO}_{2}$, nanodomains are formed within the well known microdomain structures. In the images, these nanodomains are seen as stripes or tweed-like structures of varying density, since they are tilted against the electron beam. The dimensions of the nanodomains rise toward the morphotropic phase boundary. ${ }^{10}$ The orientation of the nanodomain walls could not in all experiments be unambiguously attributed to the common pseudocubic $\{101\}$ or $\{010\}$ planes, nor to the $\{211\}$ planes expected in the monoclinic phase. Investigations are still in progress.

Along with the changing morphology of the nanodomains, the intensity distribution of the x-ray pattern changes characteristically as well, indicated by the arrows in Fig. 3. Attempts to describe the data with a model of a simple mixture of the rhombohedral and tetragonal phase were not successful matching the results of the CBED investigation. The $\mathrm{x}$-ray patterns observed show an increase of the intensity that appears between the tetragonal 101 and 110 reflections as the composition of the samples approaches the morphotropic phase boundary. These peaks are interpreted as an effect of nanodomains, according to TEM investigations. Thus, this specific reflection can be taken as a fingerprint for the existence of nanodomains; the peak shape and intensity

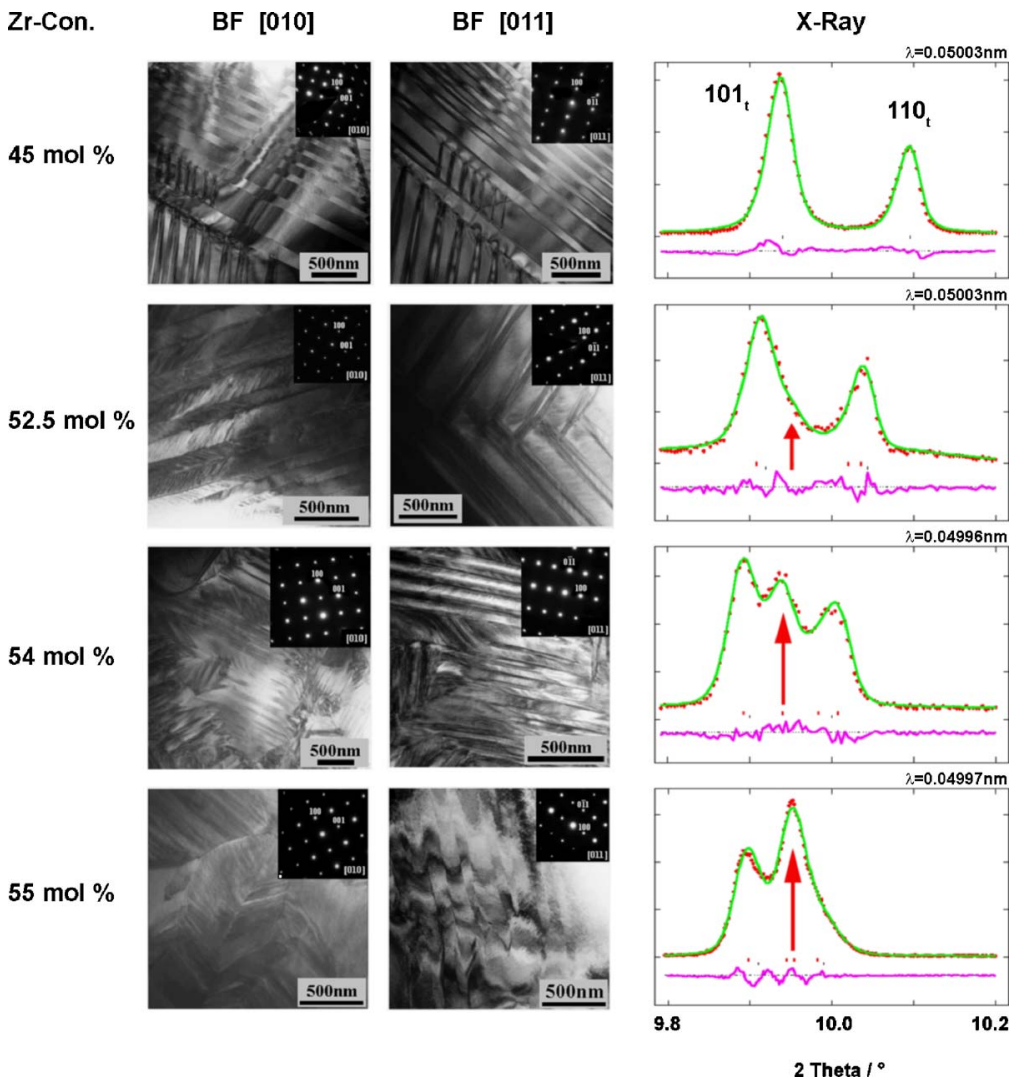

FIG. 3. (Color online) TEM bright field images (BF) along the [010] and [011] axes and corresponding sections of the x-ray full patterns of PZT ceramics with $45 \mathrm{~mol} \%, 52.5 \mathrm{~mol} \%, 54 \mathrm{~mol} \%$, and $55 \mathrm{~mol} \%$ of $\mathrm{ZrO}_{2}$ are shown. Nanodomains are found in all of these samples except the one containing $45 \mathrm{~mol} \% \mathrm{ZrO}_{2}$, which is clearly off the morphotropic region. 


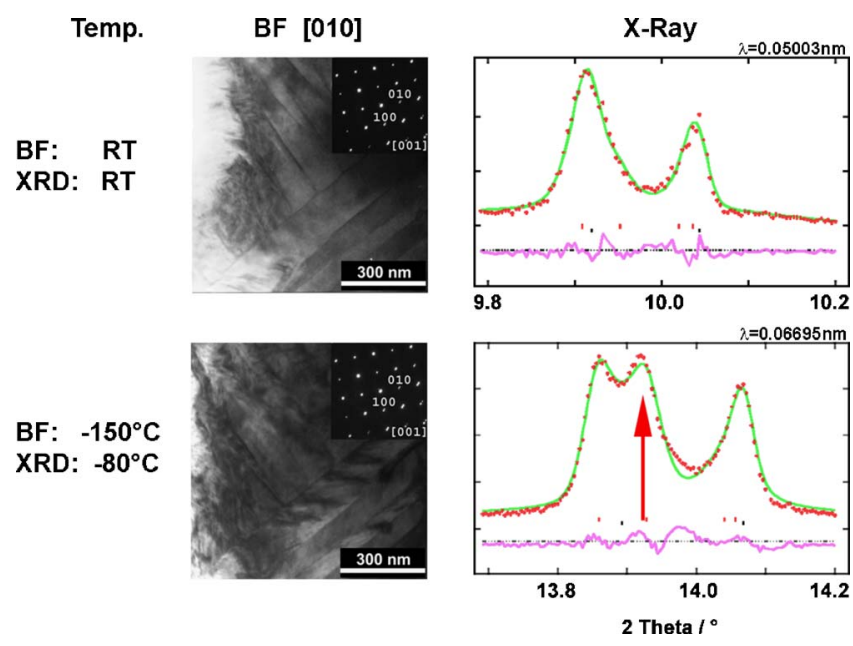

FIG. 4. (Color online) The cooling experiment of the sample with a $\mathrm{ZrO}_{2}$ content of $52.5 \mathrm{~mol} \%$ demonstrates the correlation between the nanodomain formation and the associated intensity change in the x-ray pattern. (The TEM images are of identical regions.)

are correlated with the size and amount of nanodomains present in the material. A detailed quantitative analysis of the $\mathrm{X}$-ray data is published elsewhere. ${ }^{14}$

To confirm the observed correlation between the nanodomains and the characteristic x-ray intensity, we present the observation of a cooling experiment of the $52.5 \mathrm{~mol} \% \mathrm{ZrO}_{2}$ containing sample sintered at $1050{ }^{\circ} \mathrm{C}$ (Fig. 4): At low temperatures a significantly increased amount of nanodomains is observed compared to the room temperature image. The corresponding $\mathrm{X}$-ray pattern matches the trend stated before. The joint interpretation of these experiments indicates a high correlation of the amount and the dimensions of the nanodomains and the intensity and the shape of the x-ray peaks.

Nanodomains have been found in all presented samples with compositions close to the MPB. It is concluded, that the existence of nanodomains is a characteristic feature of the
MPB region. A work of Grinberg et al. ${ }^{25}$ gives the key to understanding our experimental results. They showed, using density functional theory calculations, that the distortions of the lead ions from their ideal cubic positions are sensitive to the local zirconium and titanium environment. Since PZT is a mixed crystal, the distribution of $\mathrm{Zr}$ and $\mathrm{Ti}$ is statistical. Therefore, the polarization directions of the individual lead atoms differ from the mean polarization direction within a single microdomain. It is a matter of statistics that each domain will contain lead atoms whose local environment contradicts the mean polarization direction. We conclude that such configurations of atoms act as seeds for the formation of nanodomains, which can either already exist in the equilibrium (Fig. 3) or may form during the poling process as indicated by TEM experiments (Fig. 5 and the corresponding video).

To understand the role of the nanodomains during the poling process, an in situ TEM investigation with applied electric field perpendicular to the sample surface was carried out. Some characteristic images are presented in Fig. 5. A video of a part of the experiment can be seen as supplementary online material at the home page of this journal. The nanodomains can be identified either as stripe or tweed-like structures within the microdomains. It is observed that mainly the nanodomains respond to the electric field, while the microdomain structure remains visibly unchanged during the whole experiment.

As a complementary experiment, an in situ x-ray study with applied electric field was carried out. The specific reflection correlated with the existence of nanodomains was observed. Exemplarily, the results are shown for the samples containing $52.5 \mathrm{~mol} \%, 53 \mathrm{~mol} \%$, and $54 \mathrm{~mol} \%$ of $\mathrm{ZrO}_{2}$ (Fig. 6). The patterns vary with composition, yet the trend remains the same for all samples: As the electric field increases during the first cycle, the peak correlated with the nanodomains increases, as indicated by the black (gray) ar-
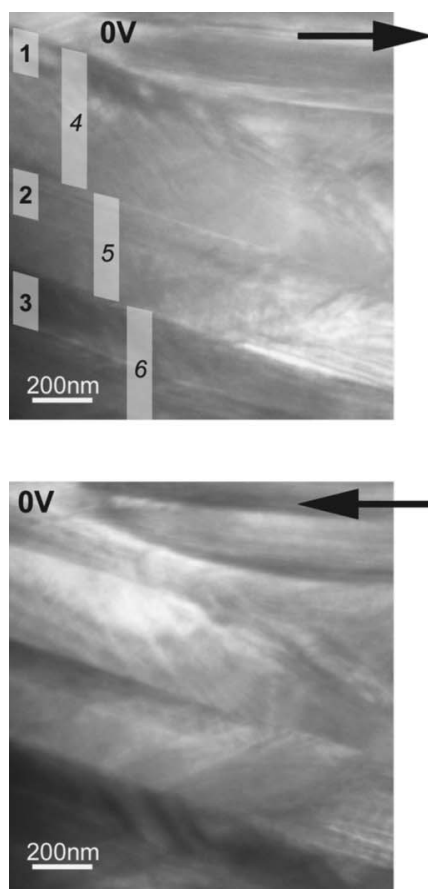
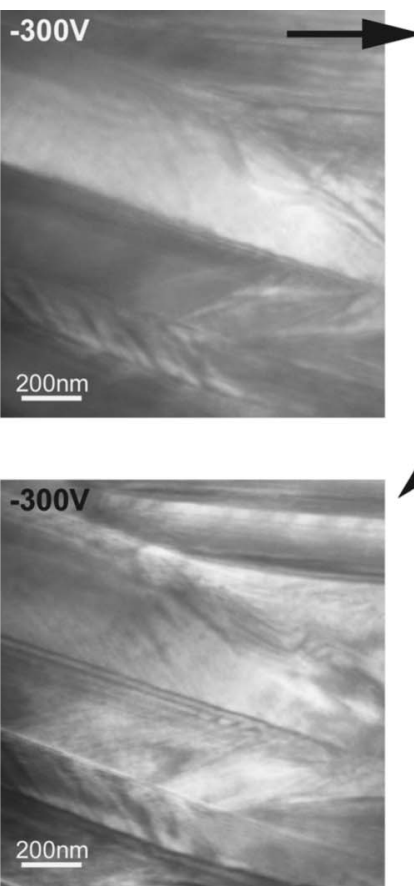
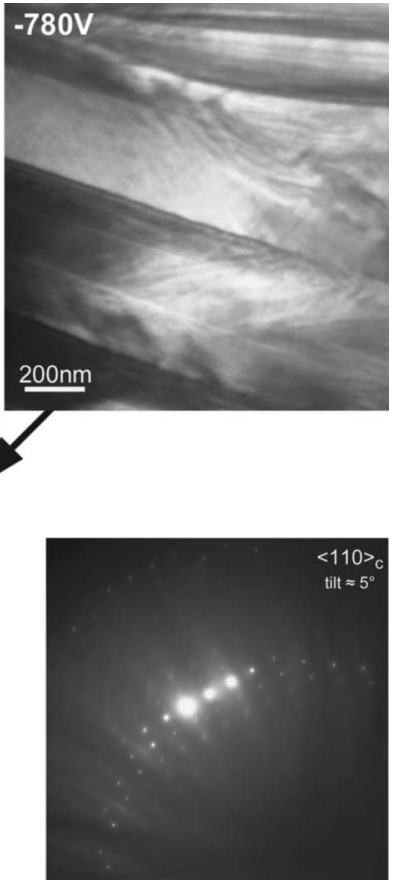

FIG. 5. TEM bright-field images taken during the third cycle of an in situ experiment with applied electric field. The sample contains $54 \mathrm{~mol} \%$ of $\mathrm{ZrO}_{2}$. The images show the microdomain and nanodomain structures; the microdomains walls are labeled with the numbers $1-3$, and the domains with 4-6 (upper left). While the microdomain structure remains visibly unchanged during the poling cycles, the nanodomains, visible as stripe-like structures within the microdomains, respond to the electric field. A complete movie of this poling cycle can be seen in the online version of the journal. The electron diffraction pattern shows that the sample is tilted approximately $5^{\circ}$ off a pseudocubic [110] axis. 


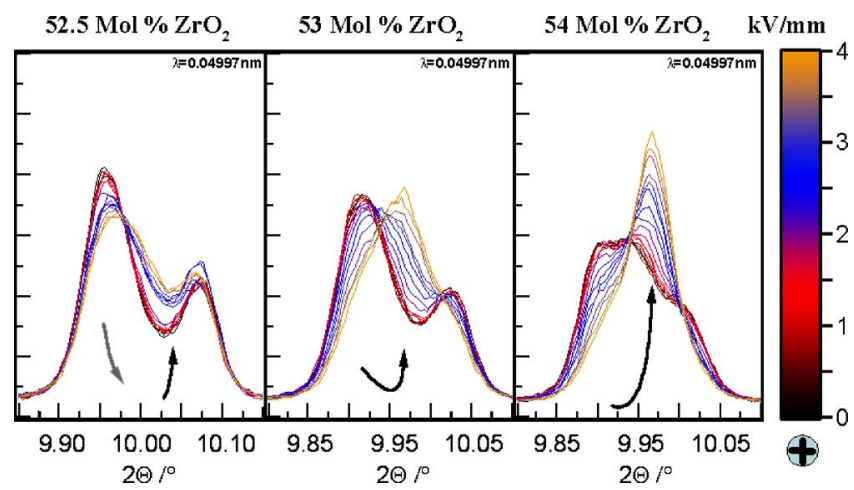

FIG. 6. (Color online) In situ high resolution x-ray diffraction (XRD) of the same significant set of reflections shown in Fig. 3. The evolution of the peak correlated with the existence of nanodomains with an applied electric field is demonstrated.

rows in Fig. 6. The black arrows indicate the evolution of the intensity maximum, as the field is applied. The black arrow for the composition $52.5 \mathrm{~mol} \%$ of $\mathrm{ZrO}_{2}$ shows the rising intensity contribution, as the maximum intensity decreases (gray arrow). The increase is most pronounced in the sample containing $54 \mathrm{~mol} \%$ of $\mathrm{ZrO}_{2}$, less pronounced in the sample containing $53 \mathrm{~mol} \%$ of $\mathrm{ZrO}_{2}$, and visible only as a broadening of the 110 peaks in the sample containing $52.5 \mathrm{~mol} \%$ of $\mathrm{ZrO}_{2}$. During further bipolar cycling, the peak correlated with the nanodomains remains present. $^{26}$

Our experimental results on the poling behavior of morphotropic PZTs are in agreement with predictions of Jin et $a l .{ }^{17}$ concerning extreme piezoelectricity in adaptive ferroelectrics with a miniaturized domain structure. They are also supported by the work of Wang et al. ${ }^{27}$ in which it was anticipated from a TEM study of the nanodomain structure of morphotropic $\mathrm{Pb}\left(\mathrm{Mg}_{1 / 3} \mathrm{Nb}_{2 / 3}\right) \mathrm{O}_{3}-\mathrm{PbTiO}_{3}$ that the nanodomains might easily respond to an external field, resulting in outstanding piezoelectric properties.

The correlation of the specific x-ray reflection and the nanodomains has been established for both, ex situ and in situ experiments with applied electric field, as well as for a temperature dependent study. Now, this correlation can be used as a tool to investigate the influence of dopants on the domain structure. Technically important PZT ceramics are usually highly doped. Lanthanum is one of the important soft $A$-site dopants. A preliminary synchrotron x-ray study on a PZT sample with $52.5 \mathrm{~mol} \% \mathrm{ZrO}_{2}$ and $1 \mathrm{~mol} \% \mathrm{La}$ doping shows an X-ray pattern very similar to the pattern of notdoped PZTs with a composition between 53 and 54 mol \% $\mathrm{ZrO}_{2}$. This indicates a significantly increased amount of nanodomains compared to the undoped sample. A possible interpretation is that the dopant acts as a seed for the formation of nanodomains. Further studies on doped samples with different compositions are in progress.

\section{CONCLUSION}

Our experimental evidence offers an interpretation of the extrinsic piezoelectric effect in PZT ceramics close to the morphotropic phase boundary. Symmetry investigations of representative domain structures by CBED close to the morphotropic phase boundary in combination with domain struc- ture analysis ${ }^{10}$ have given no indication for the coexistence of the tetragonal and the rhombohedral microdomains as the relevant feature of the microstructure. Neither an indication for the existence of homogeneous microscopic regions of monoclinic symmetry or monoclinic domain configurations was found. Instead, a miniaturization of the domain structure was observed in TEM images. The interpretation of x-ray patterns was therefore done with respect to the observed nanodomain structure, yet a correlation between nanodomains and the monoclinic phase cannot be excluded. ${ }^{14}$ The in situ TEM investigations with applied electric field show that mainly the nanodomains respond to the electric field while the microdomain structure did not change noticeably in our experiments. In situ x-ray data can be interpreted in good analogy.

Our experiments also offer an interpretation of the influence of soft $A$-site dopants, which may play a role as seeds for the formation of nanodomains. Even though we have only investigated one soft $A$-site dopant, we have established a reliable method to study the influence of other dopants on the domain structure and the poling process of ferroelectrics by the combination of in situ TEM and X-ray experiments.

\section{ACKNOWLEDGMENTS}

This work was supported by the Deutsche Forschungsgemeinschaft (DFG), SFB 595. The in situ TEM experiment with applied electric field was carried out at the Institute of Nanotechnology, Research Center Karlsruhe. We thank Professor Hahn for the possibility to use the microscope. The authors want to thank G. Schierning for critically reading the manuscript.

${ }^{1}$ P. G. Lucuta, V. Teodorescu, and F. Vasiliu, Appl. Phys. A: Solids Surf. 37, 237 (1985).

${ }^{2}$ P. G. Lucuta, J. Am. Ceram. Soc. 72, 933 (1989).

${ }^{3}$ B. G. Demczyk, R. S. Rai, and G. Thomas, J. Am. Ceram. Soc. 73, 615 (1990).

${ }^{4}$ A. G. Khachaturyan, S. M. Shapiro, and S. Semenovskaja, Phys. Rev. B 43, 10832 (1991).

${ }^{5}$ J. F. Li, X. Dai, A. Chow, and D. Viehland, J. Mater. Res. 10, 926 (1995).

${ }^{6}$ X. Dai, Z. Xu, J. F. Li, and D. Viehland, J. Mater. Res. 11, 618 (1996).

${ }^{7}$ X. Dai, Z. Xu, J. F. Li, and D. Viehland, J. Mater. Res. 11, 626 (1996).

${ }^{8}$ Y. M. Jin, Y. U. Wang, and A. G. Khachaturyan, J. Appl. Phys. 94, 3629 (2003).

${ }^{9}$ D. I. Woodward, J. Knudsen, and I. M. Reaney, Phys. Rev. B 72, 104110 (2005).

${ }^{10}$ L. A. Schmitt, K. A. Schönau, R. Theissmann, H. Fuess, H. Kungl, and M. J. Hoffmann, J. Appl. Phys. 101, 074107 (2007).

${ }^{11}$ W. Cao and E. Cross, Phys. Rev. B 47, 4825 (1993).

${ }^{12}$ B. Noheda, D. E. Cox, G. Shirane, J. A. Gonzalo, L. E. Cross, and S.-E. Park, Appl. Phys. Lett. 74, 2059 (1999).

${ }^{13}$ Y. M. Jin, Y. U. Wang, A. G. Khachaturyan, J. F. Li, and D. Viehland, Phys. Rev. Lett. 91, 197691 (2003).

${ }^{14}$ K. A. Schönau, L. A. Schmitt, M. Knapp, H. Fuess, R.-A. Eichel, H. Kungl, and M. J. Hoffmann, Phys. Rev. B 75(18), 184117 (2007).

${ }^{15}$ Y. U. Wang, Phys. Rev. B 74, 104109 (2006).

${ }^{16}$ H. Boysen, Z. Kristallogr. 220, 726 (2005).

${ }^{17}$ Y. M. Jin, Y. U. Wang, A. G. Khachaturyan, J. F. Li, and D. Viehland, J. Appl. Phys. 94, 3629 (2003).

${ }^{18}$ Y. U. Wang, Phys. Rev. B 73, 014113 (2006).

${ }^{19}$ I. MacLaren, L. A. Schmitt, H. Fuess, H. Kungl, and M. J. Hoffmann, J. Appl. Phys. 97, 094102 (2005). 
${ }^{20}$ M. Hammer and M. J. Hoffmann, J. Am. Ceram. Soc. 81, 721 (1998).

${ }^{21}$ M. Knapp, C. Baehtz, H. Ehrenberg, and H. Fuess, J. Synchrotron Radiat. 11, 328 (2004).

${ }^{22}$ M. Knapp, V. Joco, C. Baehtz, H. H. Brecht, A. Berghaeuser, H. Ehrenberg, H. von Seggern, and H. Fuess, Nucl. Instrum. Methods Phys. Res. A 521, 565 (2004).

${ }^{23}$ B. Jaffe, W. R. Cook, and H. Jaffe, Piezoelectric Ceramics (Academic
Press, London, 1971).

${ }^{24}$ G. Arlt, J. Math. Sci. 25, 2655 (1990).

${ }^{25}$ I. Grinberg, V. R. Cooper, and A. M. Rappe, Nature 419, 909 (2002).

${ }^{26}$ K. A. Schönau, M. Knapp, H. Fuess, H. Kungl, and M. J. Hoffmann, Phys. Rev. B (to be published).

${ }^{27}$ H. Wang, J. Zhu, N. Lu, A. A. Bokov, Z.-G. Ye, and X. W. Zhang, Appl. Phys. Lett. 89, 042908 (2006). 